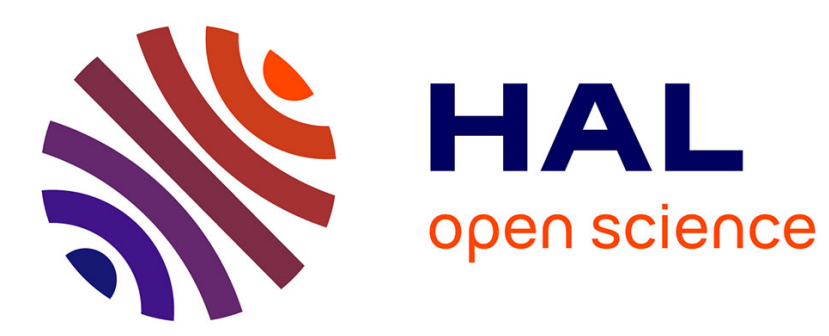

\title{
Fractional central pattern generators for bipedal locomotion
}

\author{
Carla M. A. Pinto, J. A. Tenreiro Machado
}

\section{To cite this version:}

Carla M. A. Pinto, J. A. Tenreiro Machado. Fractional central pattern generators for bipedal locomotion. Nonlinear Dynamics, 2010, 62 (1-2), pp.27-37. 10.1007/s11071-010-9696-4 . hal-00582637

\section{HAL Id: hal-00582637 \\ https://hal.science/hal-00582637}

Submitted on 3 Apr 2011

HAL is a multi-disciplinary open access archive for the deposit and dissemination of scientific research documents, whether they are published or not. The documents may come from teaching and research institutions in France or abroad, or from public or private research centers.
L'archive ouverte pluridisciplinaire HAL, est destinée au dépôt et à la diffusion de documents scientifiques de niveau recherche, publiés ou non, émanant des établissements d'enseignement et de recherche français ou étrangers, des laboratoires publics ou privés. 


\title{
Fractional Central Pattern Generators for Bipedal Locomotion
}

\author{
Carla M.A. Pinto \\ Centro de Matemática da Universidade do Porto and \\ Departament of Matemathics, Institute of \\ Engineering of Porto, Rua Dr. Antonio Bernardino de \\ Almeida, 4200-072 Porto, Portugal \\ e-mail: cap@isep.ipp.pt
}

$\&$

\author{
Department of Electrical Engineering, Institute of \\ Engineering of Porto, Rua Dr. Antonio Bernardino de \\ Almeida, 4200-072 Porto, Portugal \\ e-mail: jitm@isep.ipp.pt
}

\begin{abstract}
Locomotion has been a major research issue in the last few years. Many models for the locomotion rhythms of quadrupeds, hexapods, bipeds and other animals have been proposed. This study has also been extended to the control of rhythmic movements of adaptive legged robots.

In this paper, we consider a fractional version of a central pattern generator (CPG) model for locomotion in bipeds. A fractional derivative $D^{\alpha} f(x)$, with $\alpha$ non-integer, is a generalization of the concept of an integer derivative, where $\alpha=1$. The integer CPG model has been proposed by Golubitsky, Stewart, Buono and Collins, and studied later by Pinto and Golubitsky. It is a network of four coupled identical oscillators and has dihedral symmetry. We study parameter regions where periodic solutions, identified with legs' rhythms in bipeds, occur, for $0<\alpha \leq 1$. We find that the amplitude and the period of the periodic solutions, identified with biped rhythms, increase as $\alpha$ varies from near 0 to values close to one.
\end{abstract}

\section{Introduction}

A CPG is a network of neurons located somewhere in the nervous system capable of producing the rhythms associated to different animal gaits (Grillner, 1975).

Mathematically, CPGs are modeled as networks of identical systems of differential equations, where the individual systems model (collections of) neurons (or cells) (Collins \& Stewart, 1993a; Collins \& Stewart, 1993b; Collins \& Stewart, 1994; Kopell \& Ermentrout, 1988; Golubitsky et al, 1998; Golubitsky et al, 1999).

These CPG models have been studied in animal locomotion and, more recently, in the field of robotics.

Golubitsky, Stewart, Buono and Collins (Golubitsky et al, 1998; Golubitsky et al, 1999) propose models for locomotor CPGs for $2 n$-legged animals, for all values of $n \in \mathfrak{N}$. These networks consist of $4 n$ coupled identical cells, arranged in two cycles of $2 n$ cells, that are able to generate the phase relations seen in the animal gaits. These phase relations are a result of the symmetry of the model. The model 
presents the bilateral symmetry of animals and a cyclic translational symmetry, from back to front, that is, cell $4 n$ is coupled to cell 2 , and the same applies for cells $4 n-1$ and 1 . Similar network architectures also generate locomotor patterns of legless animals, such as worms, snakes and lampreys. In those locomotor networks, the term "leg" should be interpreted as "muscular unit". Unwanted conjugacies presented in previous CPG models for animal gaits, such as the one between trot and pace in the four-cells CPG models for quadrupeds (Collins \& Stewart, 1993), are prevented by this new network architecture. These new structures for CPG models are derived assuming that each joint receives signals from two neurons. This reasoning draws an analogy with muscular action in real joints, since two muscle groups, flexors and extensors, control most joints. Thus, abstractly, CPG models control muscle groups rather than legs and minimal CPG networks should have two cells per leg.

In the field of robotics, most models for CPG locomotor patterns of robots have one oscillator per joint, and consequently, each joint is driven by two neurons (Taga et al, 1991; Liu et al, 2008; Baydin, 2008). There are a few authors though that, similarly to Collins and Stewart (Collins \& Stewart, 1993), assume that locomotor CPGs have only one cell per limb (Righetti et al, 2006; Righetti \& Ijspeert, 2008). The main disadvantage, as explained previously for the four-cells CPG networks of Collins and Stewart, is that it is necessary to change the architecture of the network in order to observe all of the animal movements in the robot. The advantage of this approach is that only half of the oscillators are needed to control the motor patterns, comparatively with the CPG networks proposed by Golubitsky et al (Golubitsky et al, 1998; Golubitsky et al, 1999), and, therefore, less parameter values need to be recomputed in gait transition.

CPG-like coupled cell systems are able to produce sustained rhythmic activation patterns even when isolated from external stimuli. CPGs have other nice properties, such as limit cycle behaviour, phase locking modes, robustness against small perturbations and smooth online modulation of trajectories. All these characteristics can be achieved through changes in parameter values of the equations (Marder \& Bucher, 2001; Matos et al, 2009, Righetti et al, 2006). These features make CPGs an attractive option to model the control of legged robot locomotion for bipedal, quadrupedal and other designs (Marder \& Bucher, 2001; Matos et al, 2009, Righetti et al, 2006, Righetti \& Ijspeert, 2008). In Matos et al (Matos et al, 2009), it is proposed a bio-inspired robotic controller able to generate locomotion and to easily switch between different types of gaits. The duty factor and inter limb phase values are adjusted according to the velocities. This improves stability and response of the robot during locomotion. Degallier et al (Degallier et al, 2006) use a dynamical systems' approach yielding the online generation trajectory in a robot performing a drumming task. These trajectories have both rhythmic and discrete parts. Righetti et al (Righetti et al, 2008) designed a CPG architecture based on coupled dynamical systems where gait transition is achieved by parameter change and where sensory feedback is included. Righetti and Ijspeert (Righett \& Ijspeert, 2006) use symmetric coupled systems theory (Golubitsky \& Stewart, 2006) to design a generic coupling architecture for a CPG and showed how it could be applied to the control of a crawling humanoid robot.

To conclude, it is also demonstrated that CPG-like controllers can be successfully implemented as analog electronic circuits (Lewis et al, 2003; Tenore et al, 2004). 
The network architecture $L E G$ for the central pattern generator for bipedal legs rhythms studied here is shown in Fig.1.

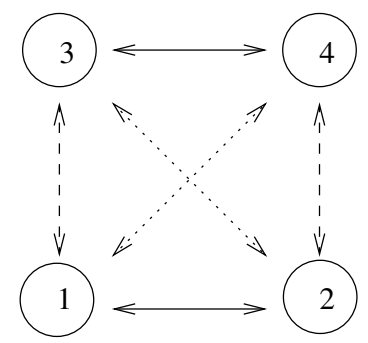

Fig. 1. CPG network LEG for the control of biped legs. Cells 1 and 3 send signals to the left leg, cells 2 and 4 send signals to the right leg (Fig 2, Pinto \& Golubitsky, 2006)

The CPG network LEG consists of four coupled identical cells. In the graph of Fig. 1, the nodes represent the cells and the arrows represent the couplings. Different arrows mean different coupling strengths. This network is capable of producing periodic solution types identified with biped locomotion rhythms, namely, walk, run, two-legged jump, two-legged hop, hesitation-walk, asymmetric hop, skip, gallop, and one-legged hop.

In this paper we consider a fractional version of the CPG LEG represented in Fig.1. We vary the order of $\alpha$ and we analyze the amplitude and period changes of periodic solutions produced by the fourcell CPG model and identified with legs' rhythms in bipeds.

\section{2.- Fractional calculus - summary}

Fractional calculus $(\mathrm{FC})$ is a generalization of the ordinary integer differentiation and integration to an arbitrary order (Oldham \& Spanier, 1974; Samko et al, 1993, Miller \& Ross, 1993). The subject was initiated in 1695 by Leibniz that sent a letter to L'Hospital with the question: "Can the meaning of derivatives with integer order be generalized to derivatives with non-integer orders?". In the last two decades we witnessed an increasing interest in the FC and relevant applications emerged in the areas of physics and engineering (Outstaloup, 1991; Mainardi, 1996, Machado , 1997; Nigmatullin, 2006; Podlubny, 1999; Tenreiro Machado, 2001; Chen \& Moore, 2002; Baleanu, 2009; Tenreiro Machado, 2009).

There are several definitions of fractional derivatives, being three of the most important the Riemann - Liouville, the Grunwald - Letnikov, and the Caputo given by:

$$
\begin{gathered}
{ }_{a} D_{t}^{\alpha} f(t)=\frac{1}{\Gamma(n-\alpha)} \frac{d^{n}}{d t^{n}} \int_{a}^{t} \frac{f(\tau)}{(t-\tau)^{\alpha-n+1}} d \tau, n-1<\alpha<n \\
{ }_{a} D_{t}^{\alpha} f(t)=\lim _{h \rightarrow 0} \frac{1}{h^{\alpha}} \sum_{k=0}^{\left[\frac{t-a}{h}\right]}(-1)^{k}\left(\begin{array}{l}
\alpha \\
k
\end{array}\right) f(t-k h)
\end{gathered}
$$




$$
{ }_{a} D_{t}^{\alpha} f(t)=\frac{1}{\Gamma(\alpha-n)} \int_{a}^{t} \frac{f^{(n)}(\tau)}{(t-\tau)^{\alpha-n+1}} d \tau, n-1<\alpha<n
$$

where $\Gamma()$ is the Euler's gamma function, $[x]$ means the integer part of $x$, and $h$ is the step time increment.

It is also possible to generalize several results based on transforms, yielding expressions such as the Laplace expression:

$$
L\left\{{ }_{0} D_{t}^{\alpha} f(t)\right\}=s^{\alpha} L\{f(t)\}-\sum_{k=0}^{n-1} s^{k}{ }_{0} D_{t}^{\alpha-k-1} f\left(0^{+}\right)
$$

where $s$ and $L$ represent the Laplace variable and operator, respectively.

These definitions demonstrate that fractional derivatives capture the history of the variable, or, by other words, have memory, contrary to integer derivatives, that are local operators.

The Grunwald - Letnikov formulation inspires the numerical calculation of the fractional derivative based on the approximation of the time increment $h$ through the sampling period $T$ and the series truncation at the $r$ th term. This method is often denoted as Power Series Expansion (PSE) yielding the equation in the $z$ - domain:

$$
Z\left\{D^{\alpha} x(t)\right\} \approx\left[\frac{1}{T^{\alpha}} \sum_{k=0}^{r} \frac{(-1)^{k} \Gamma(\alpha+1)}{k ! \Gamma(\alpha-k+1)} z^{-k}\right] X(z)
$$

where $X(z)=Z\{x(t)\}$ and $z$ and $Z$ represent the $z$-transform variable and operator, respectively.

In fact, expression (2) represents the Euler (or first backward difference) approximation in the $s \rightarrow z$ discretization scheme, being the Tustin approximation another possibility. The Euler and Tustin rational expressions, $H_{0}\left(z^{-1}\right)=\frac{1}{T}\left(1-z^{-1}\right)$ and $H_{1}\left(z^{-1}\right)=\frac{2}{T} \frac{1-z^{-1}}{1+z^{-1}} \quad$ respectively, are often called generating approximants of zero and first order, respectively. Therefore, the generalization of these conversion methods leads to the non-integer order results:

$$
\begin{aligned}
& s^{\alpha} \approx\left[\frac{1}{T}\left(1-z^{-1}\right)\right]^{\alpha}=H_{0}^{\alpha}\left(z^{-1}\right) \\
& s^{\alpha} \approx\left(\frac{2}{T} \frac{1-z^{-1}}{1+z^{-1}}\right)^{\alpha}=H_{1}^{\alpha}\left(z^{-1}\right)
\end{aligned}
$$

We can obtain a family of fractional differentiators generated by $H_{0}^{\alpha}\left(z^{-1}\right)$ and $H_{1}^{\alpha}\left(z^{-1}\right)$ weighted by the factors $p$ and $1-p$, yielding:

$$
s_{0}^{\alpha} \approx p H^{\alpha}\left(z^{-1}\right)+(1-p) H_{1}^{\alpha}\left(z^{-1}\right)
$$

In order to get a rational expression, the final approximation corresponds to a PSE or a rational fraction expansion. This approach is often denoted by Continued Fraction Expansion (CFE) of order $k \in \mathfrak{N}$, based on a Padé expansion in the neighborhood of $z^{-1}=0$, yielding: 


$$
H_{k}\left(z^{-1}\right)=\frac{\sum_{i=0}^{k} a_{i} z^{-i}}{\sum_{i=0}^{k} b_{i} z^{-i}}, a_{i}, b_{i} \in \mathfrak{R}
$$

Since one parameter is linearly dependent, usually it is established that $b_{0}=1$.

\section{CPG model for bipeds - review}

In this chapter we review the work of Pinto and Golubitsky (Pinto \& Golubitsky, 2006) for the CPG model for legs rhythms in bipeds. We state the general class of differential equations corresponding to $L E G$ and we review the so-called $H / K$ theory (Golubitsky \& Stewart, 2002) that helps to identify periodic solutions produced by CPG LEG with known biped locomotion patterns.

\subsection{CPG $L E G$ - equations and symmetries}

The class of ordinary differential equations (ODEs) governing the CPG model in Fig.1 is the following:

$$
\begin{aligned}
& \dot{x}_{1}=F\left(x_{1}, x_{2}, x_{3}, x_{4}\right) \\
& \dot{x}_{2}=F\left(x_{2}, x_{1}, x_{4}, x_{3}\right) \\
& \dot{x}_{3}=F\left(x_{3}, x_{4}, x_{1}, x_{2}\right) \\
& \dot{x}_{4}=F\left(x_{4}, x_{3}, x_{2}, x_{1}\right)
\end{aligned}
$$

where $x_{i} \in \Re^{k}$ is the cell $i=1,2,3,4$ variables, $k \in \mathbb{N}$ is the dimension of the cells' internal dynamics, and $F:\left(\Re^{k}\right)^{4} \rightarrow \Re^{k}$ is an arbitrary mapping. As all cells are assumed to be identical then $F$ must be the same for all cells.

The coupled cell system in (9) has $D_{2}$-symmetry. Consider the transposition that switches muscle groups between legs, we define it as $\rho=(12)(34)$. The other permutation that the coupled system (9) allows is the switch between muscle groups in each leg. Define the later as $\tau=(13)(24)$. These two independent permutations generate the symmetry group of order 4, denoted as $D_{2}$ and given by:

$$
D_{2}=Z_{2}(\rho) \times Z_{2}(\tau)
$$

This CPG model LEG is capable of producing eight periodic solutions corresponding to the bipedal gaits of two-legged hop, two-legged jump, walk, run, one-legged hop, hesitation walk, gallop, skip, and asymmetric hop. See Pinto and Golubitsky (Pinto \& Golubitsky, 2006), for the reasoning of this identification.

\subsection{The $H / K$ theory}

The identification of the periodic solutions of the network model $L E G$ with biped leg rhythms is done using symmetry arguments. The $H / K$ theorem (Golubitsky \& Stewart, 2002) uses the symmetry 
group of a network to enumerate those pairs of spatiotemporal symmetry subgroups $H$ and $K$ that can correspond to periodic solutions of differential equations associated to the network. These solutions are then identified with known biped locomotion patterns.

Let $\Gamma$ be a finite group of symmetries of the following systems of ODEs:

$$
\dot{x}=f(x)
$$

Let $x(t)$ be a periodic solution of system (11), with period normalized to 1, and let $\gamma \in \Gamma$. By symmetry, we know that $x(t)$ is also a solution to (11). We consider two cases, either both solutions intersect (thus, are identical) or the two trajectories do not intersect. If both solutions are identical, then by uniqueness of solutions there is $\theta \in S^{1}$ such that

$\chi x(t)=x(t-\theta)$, or $\gamma x(t+\theta)=x(t)$, and we say that $x(t)$ has spatiotemporal symmetry $\gamma \in \Gamma$, where $\theta$ is the temporal phase shift that corresponds to $\gamma$. If $\theta=0$ then $\gamma$ is a spatial symmetry.

Define $K$ as the subgroup of all spatial symmetries and $H$ as the subgroup of all spatiotemporal symmetries. Mathematically, we have:

$$
\begin{aligned}
& K=\{\gamma \in \Gamma: \not x(t)=x(t), \forall t\} \\
& H=\{\gamma \in \Gamma: \gamma\{x(t)\}=\{x(t)\}, \forall t\}
\end{aligned}
$$

In what follows, we discuss how symmetries of periodic solutions ( $H$ and $K$ ), in the CPG model LEG, can correspond to the biped gaits of walk and run. A similar reasoning applies to the other bipedal gaits.

Walk and run are gaits in which the two legs move half period out-of-phase. Nevertheless, these are different gaits. In the walk, muscle groups in each leg move like a pendulum and in the run, these muscles move in unison (Mann, 1982; Mann et al, 1986). These differences can be explained by their symmetry group pairs $(H, K)$. Let $\tau$ be the permutation that switches muscle groups between legs in bipeds. Applying $\tau$ to the run does not change that gait since muscles inside each leg receive the same signal. Applying $\tau$ to a walk does change the gait, in the sense that the muscle groups inside each leg receive signals that are now a half-period out of phase from the signals they received before. The later implies that the two legs are also half-period out of phase from where they were before applying $\tau$. The permutation $\tau$ is called a spatial symmetry for the run (the phase shift is zero) and is called a spatiotemporal symmetry for the walk (the phase shift is $1 / 2$ ). More precisely, applying $\tau$ to a walk and then performing a half-period phase shift does not change the walk gait. Consider now the permutation $\rho \tau$. Applying $\rho \tau$ to a walk does not change the gait, since pairs of diagonal muscles receive the same signal and the two pairs of signals are still half-period out of phase. Whereas applying symmetry $\rho \tau$ to a run results in the same gait but shifted by one half-period. Thus, $\rho \tau$ is a spatial symmetry of the walk and a spatiotemporal symmetry of the run.

For $H$ and $K$ to correspond to symmetries of periodic solutions of CPG LEG, there are some algebraic properties to be satisfied, namely, $H / K$ must be a cyclic group (Pinto \& Golubitsky, 2006, and references therein). 
To conclude, we note that the $H / K$ theory may be used to infer the CPG structure, for locomotion of $2 n$-legged animals, from the desired types of periodic solutions. Moreover, Buono \& Golubitsky (Buono \& Golubitsky, 2001) have used this mathematical tool to prove that there is only one CPG model with eight cells capable of producing periodic solution types identified with the quadruped walk, trot and pace.

\subsection{Results and discussion}

We simulate the fractional version of the coupled cell system (9), given by:

$$
\begin{aligned}
& D^{\alpha} x_{1}=F\left(x_{1}, x_{2}, x_{3}, x_{4}\right) \\
& D^{\alpha} x_{2}=F\left(x_{2}, x_{1}, x_{4}, x_{3}\right) \\
& D^{\alpha} x_{3}=F\left(x_{3}, x_{4}, x_{1}, x_{2}\right) \\
& D^{\alpha} x_{4}=F\left(x_{4}, x_{3}, x_{2}, x_{1}\right)
\end{aligned}
$$

where $D^{\alpha}$, with $\alpha$ non-integer, is a generalization of the concept of an integer derivative, where $\alpha=1$.

We consider the Morris-Lecar equations (Morris \& Lecar, 1981) as internal cell dynamics. The coupling is linearly diffusive. The nondimensionalized Morris-Lecar equations (Rinzel \& Ermentrout, 1989) are a system of two ordinary differential equations given by:

$$
\begin{aligned}
& \dot{\mathrm{v}}=-\mathrm{g}_{\mathrm{Ca}} \mathrm{m}(\mathrm{v})(\mathrm{v}-1)-\mathrm{g}_{1}\left(\mathrm{v}-\mathrm{v}_{1}\right)-\mathrm{g}_{\mathrm{k}} \mathrm{w}\left(\mathrm{v}-\mathrm{v}_{\mathrm{k}}\right)+\mathrm{i}=\mathrm{f}(\mathrm{v}, \mathrm{w}) \\
& \dot{\mathrm{w}}=\phi \tau(\mathrm{v})(\mathrm{n}(\mathrm{v})-\mathrm{w})=\mathrm{g}(\mathrm{v}, \mathrm{w})
\end{aligned}
$$

where $m(v)=\frac{1}{2}\left(1+\tanh \left(\frac{v-v_{1}}{v_{2}}\right)\right), n(v)=\frac{1}{2}\left(1+\tanh \left(\frac{v-v_{3}}{v_{4}}\right)\right), \tau(v)=\cosh \left(\frac{v-v_{3}}{2 v_{4}}\right)$. Each term $\mathrm{g}_{\mathrm{Ca}} \mathrm{m}(\mathrm{v})(\mathrm{v}-1)$, $\mathrm{g}_{1}\left(\mathrm{v}-\mathrm{v}_{1}\right)$ and $-\mathrm{g}_{\mathrm{k}} \mathrm{w}\left(\mathrm{v}-\mathrm{v}_{\mathrm{k}}\right)$ models an ionic channel that regulates the voltage $\mathrm{v}$ along the membrane of the axon. Parameter i is the current stimulus.

We adopt the PSE method for the approximation of the fractional derivative in the discrete time numerical integration. However, several experiments demonstrated that it was required a slight adaption to the standard approach based on a simple truncation of the series. In fact, since our objective is to generate limit cycles, the truncation corresponds to a diminishing of the gain (Tenreiro Machado, 2009) and, consequently, leads to difficulties in the promotion of periodic orbits. Therefore, in order to overcome this limitation, we decided to include a gain adjustment factor corresponding to the sum of the truncated series coefficients. In this line of thought, since the sum of the coefficients from $k=1$ up to $k=\infty$ must be 1.0 (Tenreiro Machado, 2009), the fractional derivative approximation adopted in the sequel is: 


$$
Z\left\{D^{\alpha} x(t)\right\} \approx\left(\sum_{k=1}^{r} \frac{(-1)^{k} \Gamma(\alpha+1)}{k ! \Gamma(\alpha-k+1)}\right)^{-1}\left[\frac{1}{T^{\alpha}} \sum_{k=0}^{r} \frac{(-1)^{k} \Gamma(\alpha+1)}{k ! \Gamma(\alpha-k+1)} z^{-k}\right] X(z)
$$

The dynamics governing the fractional CPG model LEG is given by:

$$
\begin{aligned}
& x_{1}(k+1)=H\left(x_{1}(k)\right)+\Delta t^{\alpha}\left(f\left(x_{1}(k), y_{1}(k)\right)-\mathrm{k}_{21}\left(x_{1}(k)-x_{2}(k)\right)-k_{11}\left(x_{1}(k)-x_{3}(k)\right)-k_{31}\left(x_{1}(k)-x_{4}(k)\right)\right) \\
& y_{1}(k+1)=y_{1}(k)+\Delta t^{\alpha} g\left(x_{1}(k), y_{1}(k)\right) \\
& x_{2}(k+1)=H\left(x_{2}(k)\right)+\Delta t^{\alpha}\left(f\left(x_{2}(k), y_{2}(k)\right)-\mathrm{k}_{22}\left(x_{2}(k)-x_{1}(k)\right)-k_{11}\left(x_{2}(k)-x_{4}(k)\right)-k_{31}\left(x_{2}(k)-x_{3}(k)\right)\right) \\
& y_{2}(k+1)=y_{2}(k)+\Delta t^{\alpha} g\left(x_{2}(k), y_{2}(k)\right) \\
& x_{3}(k+1)=H\left(x_{3}(k)\right)+\Delta t^{\alpha}\left(f\left(x_{3}(k), y_{3}(k)\right)-\mathrm{k}_{21}\left(x_{3}(k)-x_{4}(k)\right)-k_{12}\left(x_{3}(k)-x_{1}(k)\right)-k_{32}\left(x_{3}(k)-x_{2}(k)\right)\right) \\
& y_{3}(k+1)=y_{3}(k)+\Delta t^{\alpha} g\left(x_{3}(k), y_{3}(k)\right) \\
& x_{4}(k+1)=H\left(x_{4}(k)\right)+\Delta t^{\alpha}\left(f\left(x_{4}(k), y_{4}(k)\right)-\mathrm{k}_{22}\left(x_{4}(k)-x_{3}(k)\right)-k_{12}\left(x_{4}(k)-x_{2}(k)\right)-k_{32}\left(x_{4}(k)-x_{1}(k)\right)\right) \\
& y_{4}(k+1)=y_{4}(k)+\Delta t^{\alpha} g\left(x_{4}(k), y_{4}(k)\right)
\end{aligned}
$$

where $k_{i j} \in \mathfrak{R}$ are the coupling constants and $\Delta t$ is the time increment.

The parameter values of the Morris-Lecar equations, in the numerical simulations, are chosen to be $v_{1}=0.1, \quad v_{2}=0.4, \quad v_{3}=0.3, \quad v_{4}=0.2, \quad g_{l}=0.6, \quad g_{k}=1.8, \quad v_{l}=-1.8, \quad v_{k}=0.8$ and $i=1$.

In Tables 1 and 2, we show initial conditions and parameter values for the periodic orbits, identified with the biped gaits of walk (Figures 2-4), two-legged jump (Figures 5-7), and run (Figures 8-10). Each simulation was executed until a stable periodic solution was found. Moreover, it was considered $\alpha \in] 0,1]$ and, during the numerical experiments, were evaluated cases in steps of $\Delta \alpha=0.01$. We compute the amplitude and the period of the solutions of system (16), for each value of $\alpha$ leading to Figures 11-16. We find that, for all gaits, both amplitude and period values increase as $\alpha$ goes from near 0 to values close to 1 . We also observe that, in the case of the periodic solution identified with the walk, the interval where the amplitude is greater than zero is larger than the interval for the other two gaits. This is a result of the initial condition used to simulate the corresponding periodic solution. We simulate another periodic solution identified with the walk, for an initial condition of the same order than those used here for the run and for the two-legged jump (Table 1). We find that the new interval, for amplitude values greater than zero, coincides with the one for the other two gaits. Another remark is that there is a slight increase in the values of the amplitude and the period as $\alpha$ goes to values close to 1 . This is a numerical effect due to the series truncation in the approximation of the fractional derivative.

Table 1. Initial conditions for the bipedal gaits of walk, two-legged jump, and run, where $\left(x_{i}(k), y_{i}(k)\right)$ represents the initial condition of cell $i$ at sample $k$.

\begin{tabular}{|l|l}
\hline Gait & Initial conditions
\end{tabular}




\begin{tabular}{|c|c|}
\hline walk & $\begin{array}{l}x_{1}(1)=0.17403 ; x_{1}(2)=0.17399 ; x_{1}(3)=0.17394 ; x_{1}(4)=0.17389 ; x_{1}(5)=0.17385 \\
y_{1}(1)=0.23989 ; y_{1}(2)=0.23987 ; y_{1}(3)=0.23986 ; y_{1}(4)=0.23984 ; y_{1}(5)=0.23982 ; \\
x_{2}(1)=0.18709 ; x_{2}(2)=0.18715 ; x_{2}(3)=0.18719 ; x_{2}(4)=0.18725 ; x_{2}(5)=0.18729 ; \\
y_{2}(1)=0.22566 ; y_{2}(2)=0.22568 ; y_{2}(3)=0.22569 ; y_{2}(4)=0.22572 ; y_{2}(5)=0.22573 ; \\
x_{3}(1)=0.18709 ; x_{3}(2)=0.18715 ; x_{3}(3)=0.18719 ; x_{3}(4)=0.18725 ; x_{3}(5)=0.18729 ; \\
y_{3}(1)=0.22566 ; y_{3}(2)=0.22568 ; y_{3}(3)=0.22569 ; y_{3}(4)=0.22572 ; y_{3}(5)=0.22573 ; \\
x_{4}(1)=0.17403 ; x_{4}(2)=0.17399 ; x_{4}(3)=0.17394 ; x_{4}(4)=0.17389 ; x_{4}(5)=0.17385 \\
y_{4}(1)=0.23989 ; y_{4}(2)=0.23987 ; y_{4}(3)=0.23986 ; y_{4}(4)=0.23984 ; y_{4}(5)=0.23982 ;\end{array}$ \\
\hline $\begin{array}{l}\text { two-legged } \\
\text { jump }\end{array}$ & $\begin{array}{l}x_{1}(1)=0.2911 ; x_{1}(2)=0.2886 ; x_{1}(3)=0.2861 ; x_{1}(4)=0.2835 ; x_{1}(5)=0.2810 ; \\
y_{1}(1)=0.4319 ; y_{1}(2)=0.4326 ; y_{1}(3)=0.4332 ; y_{1}(4)=0.4337 ; y_{1}(5)=0.4340 ; \\
x_{2}(1)=0.2911 ; x_{2}(2)=0.2886 ; x_{2}(3)=0.2861 ; x_{2}(4)=0.2835 ; x_{2}(5)=0.2810 ; \\
y_{2}(1)=0.4319 ; y_{2}(2)=0.4326 ; y_{2}(3)=0.4332 ; y_{2}(4)=0.4337 ; y_{2}(5)=0.4340 ; \\
x_{3}(1)=0.0470 ; x_{3}(2)=0.0477 ; x_{3}(3)=0.0484 ; x_{3}(4)=0.0493 ; x_{3}(5)=0.0502 ; \\
y_{3}(1)=0.1497 ; y_{3}(2)=0.1482 ; y_{3}(3)=0.1468 ; y_{3}(4)=0.1453 ; y_{3}(5)=0.1440 ; \\
x_{4}(1)=0.0470 ; x_{4}(2)=0.0477 ; x_{4}(3)=0.0484 ; x_{4}(4)=0.0493 ; x_{4}(5)=0.0502 ; \\
y_{4}(1)=0.1497 ; y_{4}(2)=0.1482 ; y_{4}(3)=0.1468 ; y_{4}(4)=0.1453 ; y_{4}(5)=0.1440 ;\end{array}$ \\
\hline run & $\begin{array}{l}x_{1}(1)=0.2911 ; x_{1}(2)=0.2886 ; x_{1}(3)=0.2861 ; x_{1}(4)=0.2835 ; x_{1}(5)=0.2810 ; \\
y_{1}(1)=0.4319 ; y_{1}(2)=0.4326 ; y_{1}(3)=0.4332 ; y_{1}(4)=0.4337 ; y_{1}(5)=0.4340 ; \\
x_{2}(1)=0.0470 ; x_{2}(2)=0.0477 ; x_{2}(3)=0.0484 ; x_{2}(4)=0.0493 ; x_{2}(5)=0.0502 ; \\
y_{2}(1)=0.1497 ; y_{2}(2)=0.1482 ; y_{2}(3)=0.1468 ; y_{2}(4)=0.1453 ; y_{2}(5)=0.1440 ; \\
x_{3}(1)=0.2911 ; x_{3}(2)=0.2886 ; x_{3}(3)=0.2861 ; x_{3}(4)=0.2835 ; x_{3}(5)=0.2810 ; \\
y_{3}(1)=0.4319 ; y_{3}(2)=0.4326 ; y_{3}(3)=0.4332 ; y_{3}(4)=0.4337 ; y_{3}(5)=0.4340 ; \\
x_{4}(1)=0.0470 ; x_{4}(2)=0.0477 ; x_{4}(3)=0.0484 ; x_{4}(4)=0.0493 ; x_{4}(5)=0.0502 ; \\
y_{4}(1)=0.1497 ; y_{4}(2)=0.1482 ; y_{4}(3)=0.1468 ; y_{4}(4)=0.1453 ; y_{4}(5)=0.1440 ;\end{array}$ \\
\hline
\end{tabular}

Table 2. Parameter values for the bipedal gaits of walk, two-legged jump, and run, where $\Delta t$ is the time increment, and $k_{i j}$ are the coupling constants. For more information, see text.

\begin{tabular}{|l|l|l|l|l|l|l|l|l|l|}
\hline Gait & $\Delta t$ & $\phi$ & $g_{C a}$ & $k_{11}$ & $k_{12}$ & $k_{21}$ & $k_{22}$ & $k_{31}$ & $k_{32}$ \\
\hline walk & 0.0005 & 0.2 & 1.5 & 0.1 & 0.1 & -0.3 & -0.3 & 0.2 & 0.2 \\
\hline $\begin{array}{l}\text { two- } \\
\text { legged } \\
\text { jump }\end{array}$ & 0.0005 & 1.0 & 1.8 & 0.1 & 0.1 & -0.5 & -0.5 & -0.8 & -0.8 \\
\hline run & 0.0005 & 1.0 & 1.8 & 0.1 & 0.1 & 0.0 & 0.0 & -0.7 & -0.7 \\
\hline
\end{tabular}




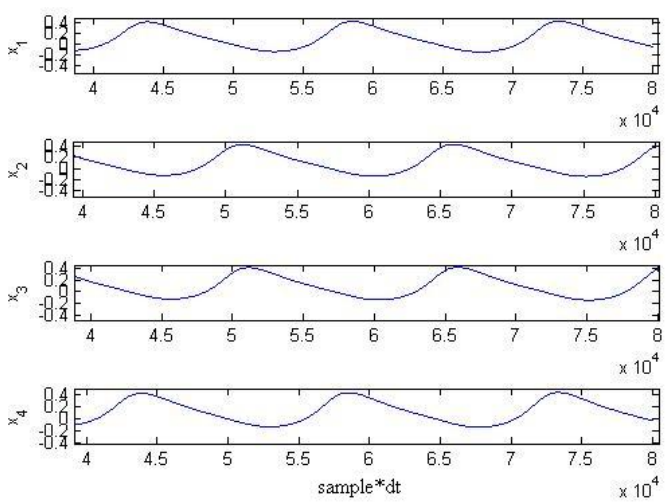

Fig. 2. Periodic solution of the CPG network LEG identified with the walk, for $\alpha=0.95$.

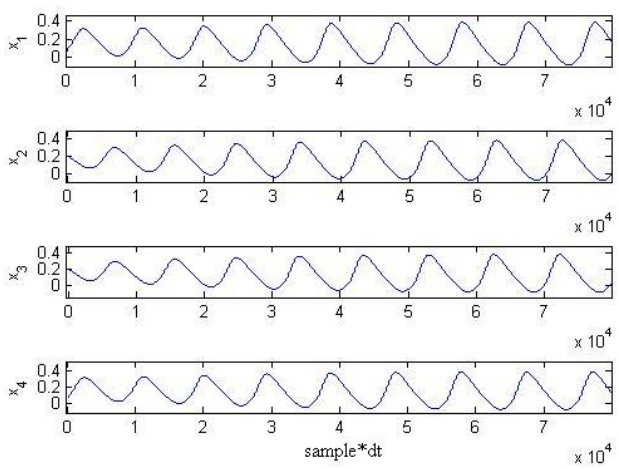

Fig. 3. Periodic solution of the CPG network LEG identified with the walk, for $\alpha=0.9$.

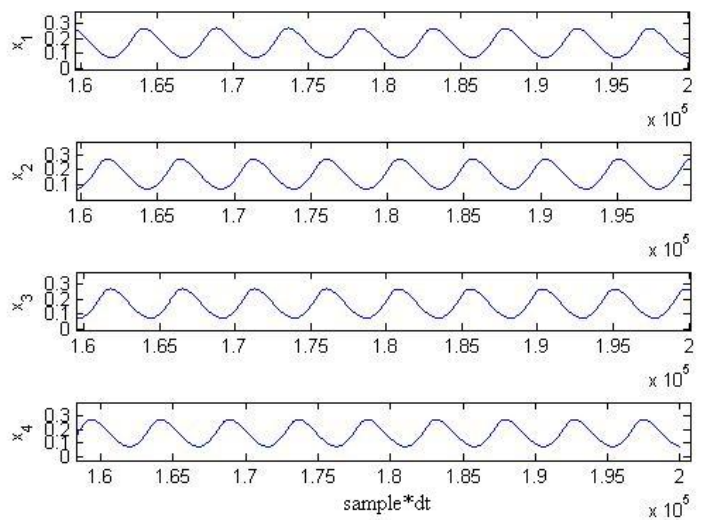

Fig. 4. Periodic solution of the CPG network LEG identified with the walk, for $\alpha=0.82$. 

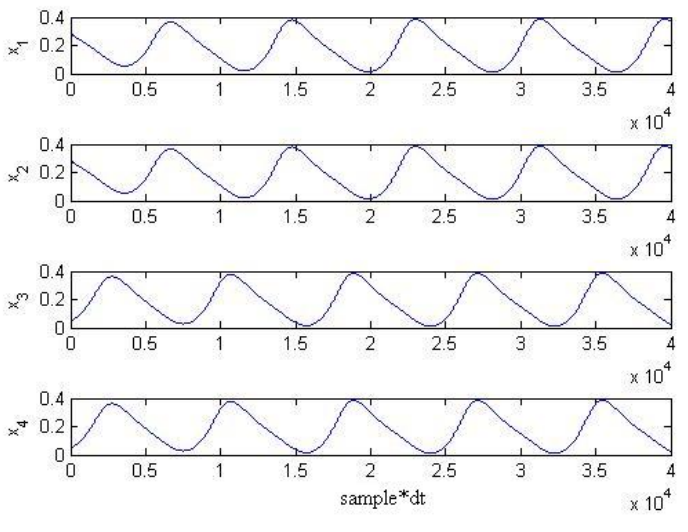

Fig. 5. Periodic solution of the CPG network LEG identified with the two-legged jump, for $\alpha=0.98$.

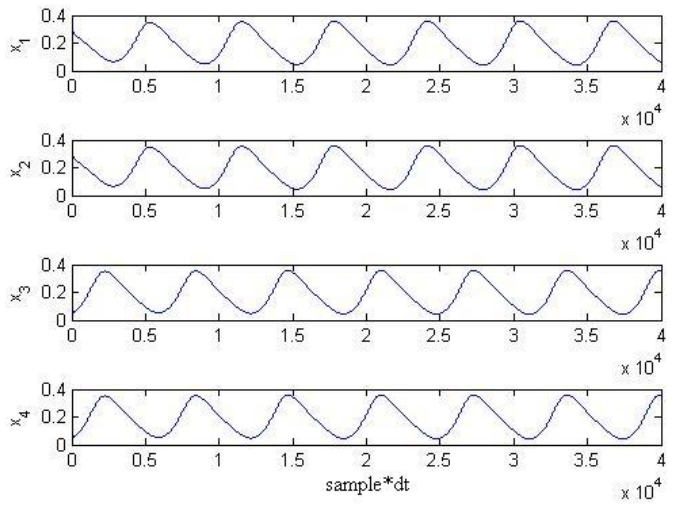

Fig. 6. Periodic solution of the CPG network LEG identified with the two-legged jump, for $\alpha=0.95$.

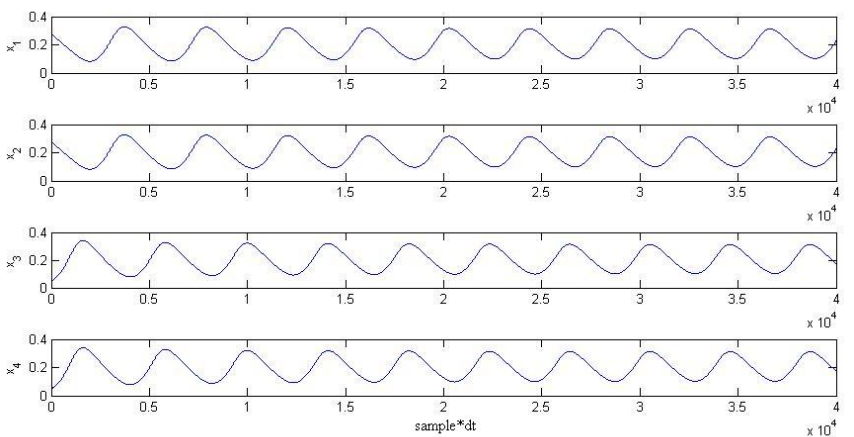

Fig. 7. Periodic solution of the CPG network LEG identified with the two-legged jump, for $\alpha=0.9$. 

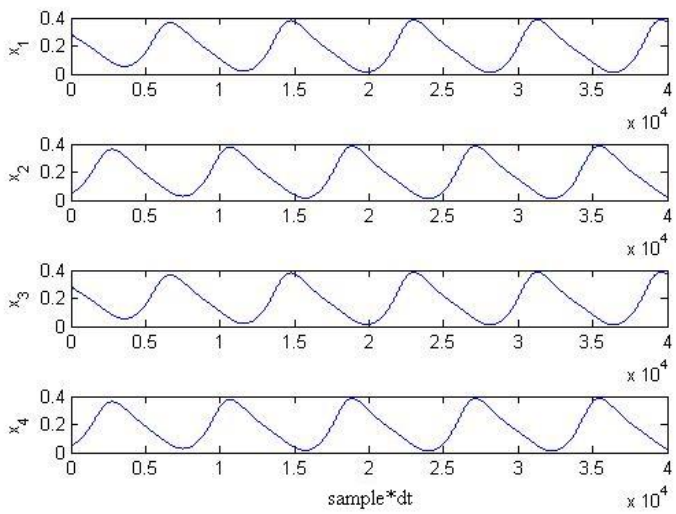

Fig. 8. Periodic solution of the CPG network LEG identified with the run, for $\alpha=0.98$.
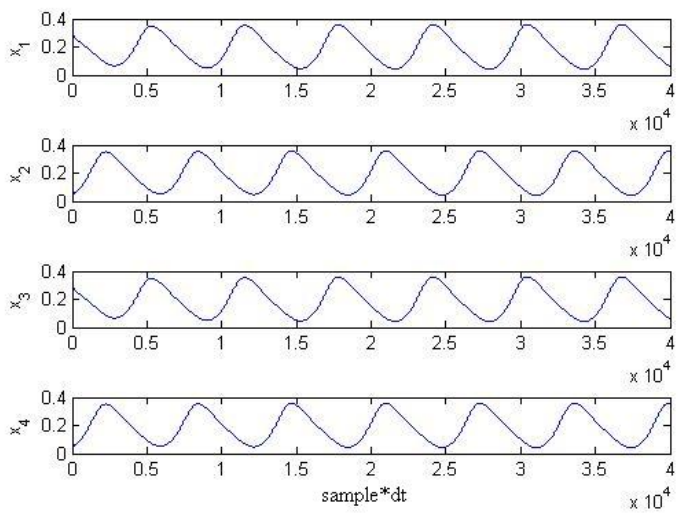

Fig. 9. Periodic solution of the CPG network LEG identified with the run, for $\alpha=0.95$.

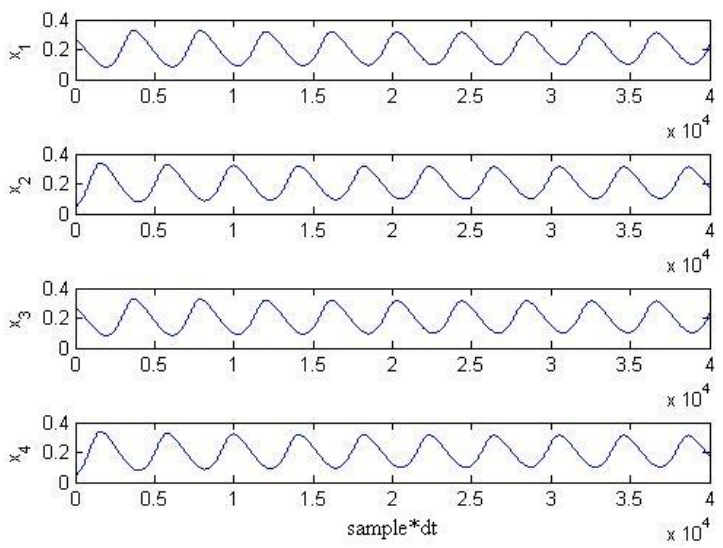

Fig. 10. Periodic solution of the CPG network LEG identified with the run, for $\alpha=0.9$. 


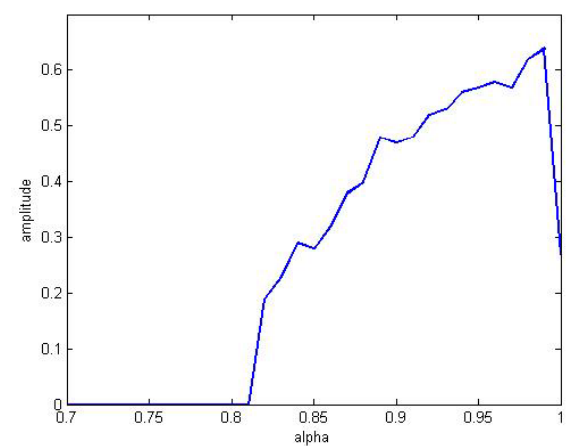

Fig. 11. Amplitude of the periodic solution produced by the CPG network $L E G$ identified with the walk, for different values of $\alpha$.

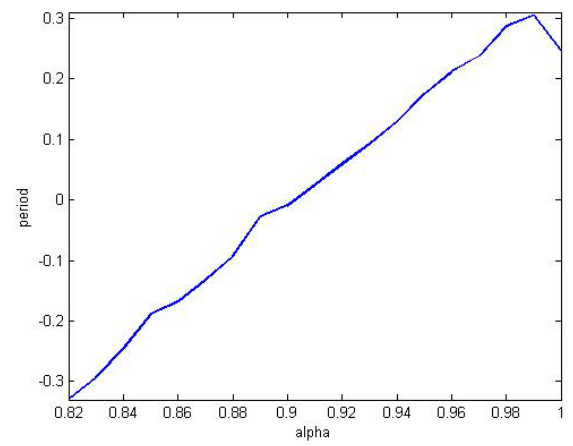

Fig. 12. Period of the periodic solution produced by the CPG network LEG identified with the walk, for different values of $\alpha$ (log scale in the vertical axis).

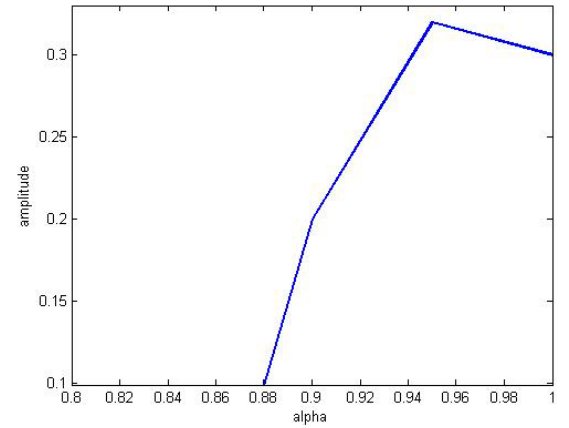

Fig. 13. Amplitude of the periodic solution produced by the CPG network LEG identified with the two-legged jump, for different values of $\alpha$. 


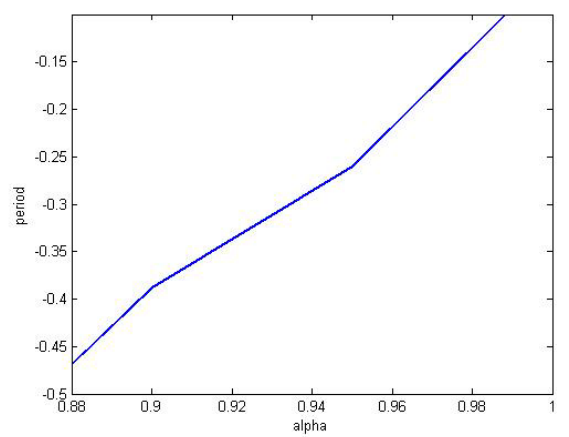

Fig. 14. Period of the periodic solution produced by the CPG network LEG identified with the twolegged jump, for different values of $\alpha$ (log scale in the vertical axis).

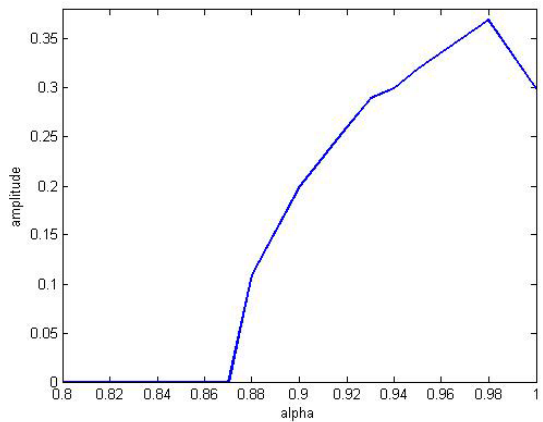

Fig. 15. Amplitude of the periodic solution produced by the CPG network LEG identified with the run, for different values of $\alpha$.

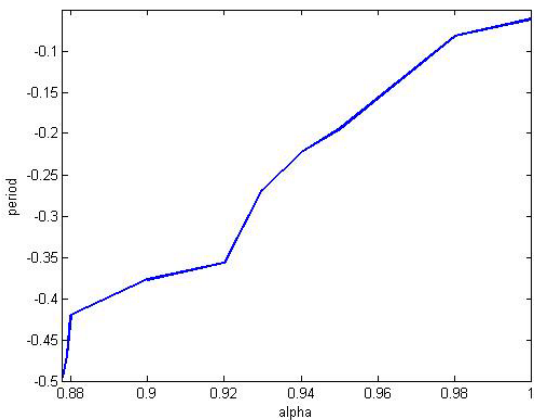

Fig. 16. Period of the periodic solution produced by the CPG network $L E G$ identified with the run, for different values of $\alpha(\log$ scale in the vertical axis).

As we can observe from Figures 11-16, $\alpha$ behaves like a bifurcation parameter, that is, as we vary $\alpha$ the dynamical behaviour of the system changes. This parameter, in particular, simplifies the work of the researcher. In fact, we do not need to know deeply the parameters of the equations, that model the dynamics of each cell, in order to increase the frequency or the amplitude of a given periodic solution, we just have to vary the value of $\alpha$. 
In Figure 17, we depict the 3D plot of the first variable of cell 1 dynamics and its derivative, for 80000 samples of the periodic solution of Figure 7.

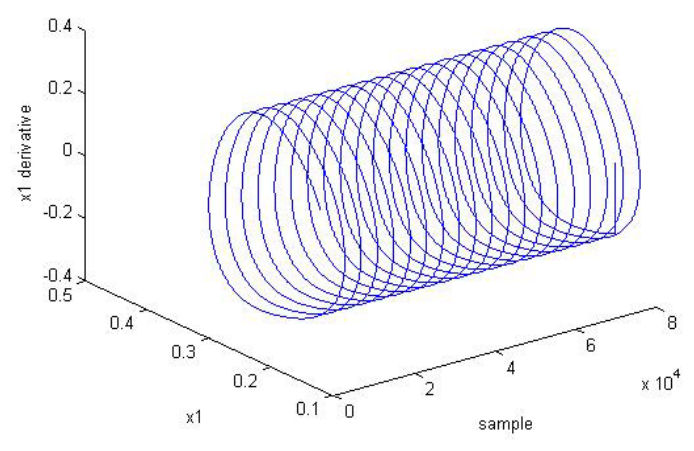

Fig. 17. 3D plot of $x_{1}$ and its derivative, for 80000 samples, for the periodic solution of Figure 7 (two-legged jump).

\section{Conclusions and future work}

We studied a fractional version of a CPG network model for legs rhythms in bipeds. We analysed the amplitude and the period values of periodic solutions, identified with three biped locomotion patterns (walk, two-legged jump, and run), for different values of $\alpha$. We found that the amplitude and the period values increase as $\alpha$ goes from values near 0 up to values close to 1 . In future work, we intend to study thoroughly the behaviour of the solutions for $0<\alpha<0.1$.

\section{Acknowledgments}

This paper was supported (in part) by Fundação para a Ciência e a Tecnologia (FCT) through the Centro de Matemática da Universidade do Porto www.fc.up.pt/cmup.

\section{References}

Baleanu, D. (2009). About fractional quantization and fractional variational principles. Communications in Nonlinear Science and Numerical Simulation, 14(6), 2520-2523.

Buono, P.L. \& Golubitsky, M. (2001) Models of central pattern generators for quadruped locomotion I. Primary gaits, J. Mathematical Biology. Vol 42, 291-326.

Chen, Y.Q. \& Moore, K.L. (2002). Discretization schemes for fractional-order differentiators and integrators. IEEE Trans. Circuits and Systems-I: Fundam. Theory Appl. 49(3), 363-367. 
Collins, J.J. \& Stewart, I. (1993a).Coupled nonlinear oscillators and the symmetries of animal gaits, J. Nonlin. Sci.,Vol. 3, pp 349-392. ISSN 1432-1467.

Collins, J.J. \& Stewart, I. (1993b). Hexapodal gaits and coupled nonlinear oscillator models. Biol. Cybern. 68, 287-298.

Collins, J.J. \& Stewart, I. (1994). A group-theoretic approach to rings of coupled biological oscillators. Biol. Cybern. 71, 95-103.

Degallier, S., Santos, C., Righetti, L. \& Ijspeert, A. (2006). Movement generation using dynamical systems: a humanoid robot performing a drumming task. IEEE-RAS International Conference on Humanoid Robots 2006.

Golubitsky, M. \& Stewart, I. (2002). The Symmetry Perspective. Progress in Mathematics, 200, Birkhauser, Basel.

Golubitsky, M. \& Stewart, I. (2006). Nonlinear dynamics of network: the groupoid formalism,. Bulletin of the American Mathematical Society, vol. 43, pp. 305-364.

Golubitsky, M., Stewart, I., Buono, P.L., \& Collins, J.J. (1998). A modular network for legged locomotion. Physica D, Vol. 115, pp 56-72. ISSN 0167-2789.

Golubitsky, M., Stewart, I., Buono, P.L., \& Collins, J.J. (1999). Symmetry in locomotor central pattern generators and animal gaits, Nature Vol. 401, pp 693-695. ISSN 0028-0836.

S. Grillner (1975). Locomotion in vertebrates: central mechanisms and reflex interaction. Physiological Reviews, Vol 55: pp 247-304.

Kopell, N., Ermentrout, J. (1988) Coupled oscillators and the design of central pattern generators. Math. Biosci. 90, 87-109.

Lewis, M.A., Etienne-Cummings, R., Hartmann, M.J., Xu, Z.R, \& Cohen, A.H. (2003). An in silico central pattern generator: silicon oscillator, coupling, entrainment, and physical computation. Biol. Cybern. Vol. 88, pp 137-151, ISSN 0340-1200.

Liu, G.L., Habib, M.K., Watanabe, K., \& Izumi, K. Central pattern generators based on Matsuoka oscillators for the locomotion of biped robots. Artif Life Rootics. Vol. 12, pp 264-269. ISSN 14335298.

Machado, J. T. (1997). Analysis and design of fractional-order digital control systems. Journal Systems Analysis, Modelling, Simulation, 27, 107-122.

Mainardi, F. (1996). Fractional relaxation-oscillation and fractional diffusion-wave phenomena. Chaos, Solitons \& Fractals, 7, 1461-1477.

Mann, R.A. (1982). Biomechanics. In Disorders of the Foot (Jahss, M.H. ed), W.B. Saunders and Co., Philadelphia, 37-67.

Mann, R.A., Moran, G.T., \& Dougherty, S.E. (1986). Comparative electromyography of the lower extremity in jogging, running and sprinting, Amer. J. Sports Med. 14, 501-510.

Matos, V., Santos, C.P. \& Pinto, C.M.A. (2009) A Brainstem-like Modulation Approach for Gait Transition in a Quadruped Robot. Proceedings of The 2009 IEEE/RSJ International Conference on Intelligent RObots and Systems, IROS 2009, St Louis, MO, USA, October 2009 
Marder, E. \& Bucher, D. (2001). Central pattern generators and the control of rhythmic movements. Current Biology, Vol. 11, Issue 23, pp 986-996, ISSN 0960-9822.

Miller, K. S., Ross, B. (1993). An introduction to the fractional calculus and fractional differential equations, John Wiley and Sons.

Morris, C. \& Lecar, H. (1981). Voltage Oscillations in the Barnacle Giant Muscle Fiber. Biophysical Journal, Vol. 35, pp 193-213.

Nigmatullin, R. (2006). The statistics of the fractional moments: Is there any chance to "read quantitatively" any randomness?. Signal Processing, 86(10), 2529-2547.

Oldham, K. B., Spanier, J. (1974). The fractional calculus: theory and application of differentiation and integration to arbitrary order. Academic Press.

Oustaloup, A. (1991). La commande CRONE: commande robuste d'ordre non entier. Hermes.

Pinto, C.M.A. \& Golubitsky, M. (2006). Central Pattern Generators for Bipedal Locomotion. Journal of Mathematical Biology, Vol. 53, No. 3, pp 474-489, ISSN 0303-6812.

Podlubny, I. (1999). Fractional-order systems and PI D -controllers. IEEE Transactions on Automatic Control, 44(1), 208-213.

Righetti, L. \& Ijspeert, A. (2006). Design methodologies for central pattern generators: an application to crawling humanoids. Proceedings of Robotics: Science and Systems, 2006, pp. 191-198.

Righetti, L, Buchli, J. \& Ijspeert, A.J. (2006). Dynamic Hebbian learning in adaptive frequency oscillators. Physica D, Vol. 216, pp 269-281. ISSN 0167-2789.

Righetti, L, \& Ijspeert, A.J. (2008). Pattern generators with sensory feedback for the control of quadruped locomotion. In IEEE International Conference on Robotics and Automation, 2008. ICRA 2008,.No 19-23, pp 819-824. ISBN 1050-4729.

Rinzel, J. \& Ermentrout, G.B. (1989) Analysis of neural excitability and oscillations. In Methods in Neuronal Modeling: From Synapses to Networks (C. Koch and I. Segev, eds) MIT Press, Cambridge, MA, 1989. ISBN 0-262-11231-0.

Samko, S. G., Kilbas, A. A., Marichev, O. I.: Fractional integrals and derivatives: theory and applications. Gordon and Breach Science Publishers (1993).

Taga, G., Yamaguchi, Y., \& Shimizu, H. (1991). Self-organized control of bipedal locomotion by neural oscillators in unpredictable environment. Biol. Cybern., Vol. 65, pp 147-169. ISSN

Tenore, F, Etienne-Cummings, R., \& Lewis, M.A. (2004) Entrainment of silicon central pattern generators for legged locomotory control. In Neural Information Processing Systems, NIPS 2003, December 8-13, 2003, Vancouver and Whistler, British Columbia, Canada. MIT Press 2004. ISBN 0-262-20152-6.

Tenreiro Machado, J. (2001). Discrete-time fractional-order controllers. Journal of Fractional Calculus \& Applied Analysis, 4, 47-66.

Tenreiro Machado, J. A. (2009). Fractional Derivatives: Probability Interpretation and Frequency Response of Rational Approximations, Communications in Nonlinear Science and Numerical Simulations, Elsevier, Volume 14, Issues 9-10, pp. 3492-3497, September-October 2009. 